\title{
Síntomas depresivos y sobrecarga en los familiares cuidadores en la enfermedad de Alzheimer: un modelo de ecuaciones estructurales
}

\author{
Vanesa Viñas-Díez, Josep Lluís Conde-Sala, Oriol Turró-Garriga, Jordi Gascón-Bayarri, Ramón Reñé-Ramírez
}

Introducción. El cuidado de los pacientes con enfermedad de Alzheimer incrementa la sobrecarga y la depresión del cuidador principal, aunque la relación entre ambas no está claramente definida.

objetivos. Explorar los factores asociados a la sintomatología depresiva y la sobrecarga en el cuidador principal y aplicar un modelo de ecuaciones estructurales para identificar la relación entre ellas.

Sujetos y métodos. La muestra estuvo formada por 127 cuidadores familiares de personas con enfermedad de Alzheimer, con un seguimiento de 24 meses. Se realizaron análisis de regresión multivariante para identificar las características de pacientes y cuidadores asociadas a la sobrecarga y la depresión del cuidador principal, y se diseñó un modelo de ecuaciones estructurales para analizar la relación entre las variables.

Resultados. En el modelo de ecuaciones estructurales, la sobrecarga tuvo un efecto directo sobre la depresión, tanto en la evaluación basal como a los 24 meses. Las variables asociadas a la sobrecarga fueron: la escolaridad y la menor salud mental del cuidador principal, y las alteraciones conductuales y la dependencia funcional del paciente. El menor nivel cognitivo del paciente y la menor salud mental del cuidador principal estuvieron asociados a la depresión. La correlación entre sobrecarga y depresión aumentó desde la evaluación basal hasta los dos años $(r=0,47$ frente a $r=0,613)$.

Conclusiones. El deterioro del paciente y la sobrecarga son factores de riesgo para la depresión en el cuidador principal. Serían necesarias intervenciones para reducir la carga y poder prevenir la depresión relacionada.

Palabras clave. Alzheimer. Cuidador. Depresión. Ecuaciones estructurales. Salud mental. Sobrecarga.

\section{Introducción}

Con el envejecimiento de la población, el número de personas con demencia aumentará progresivamente. La enfermedad de Alzheimer es el subtipo más frecuente, con un $60-80 \%$ de casos [1], y se estima que afecta a un 6,88\% de la población europea [2].

La mayoría de los pacientes diagnosticados de enfermedad de Alzheimer son asistidos principalmente por cuidadores familiares, que dedican una gran parte de su tiempo a su cuidado [3]. Esta asistencia continuada supone un esfuerzo que comporta consecuencias negativas para la salud física y mental del cuidador $[4,5]$ y crea altos niveles de sobrecarga $[6,7]$ y depresión $[8,9]$.

Diversos estudios han mostrado que algunas de las características del paciente influyen en la sobrecarga y la sintomatología depresiva del cuidador principal. Las alteraciones conductuales y la dependencia funcional se han relacionado con una mayor sintomatología en el cuidador principal [9-13]. Respecto al declive cognitivo, mientras que algunos autores consideran que no es un factor determinante de la carga o de la salud mental [14], otros estudios lo asociaban con niveles superiores de sobrecarga [15] y depresión de los cuidadores [16]. También son relevantes algunas características propias del cuidador, relacionadas con una mayor depresión y sobrecarga, como el bajo nivel educativo $[15,17]$ o la menor salud física y mental [12,18].

La sobrecarga y la sintomatología depresiva son dos respuestas típicamente ligadas al proceso de cuidado, y, aunque existe una asociación evidente entre ellas [19], su relación todavía no está bien definida. Springate y Tremont [20] expusieron que la depresión era predictora de dos de los factores que componían la sobrecarga: el impacto en la vida del cuidador y los sentimientos de culpabilidad.

Por el contrario, otros estudios señalan una relación inversa: la sobrecarga sería la responsable de los sentimientos depresivos [21-23]. Sherwood et al [24] plantearon un modelo en el que el estado mental y funcional del paciente, el sexo del cuidador principal y la relación con el paciente actuaban como predictores de la sobrecarga, y ésta, a su vez, influía sobre los síntomas depresivos del cuidador
Unidad de Demencias; Servicio de Neurología; Hospital Universitari de Bellvitge; L'Hospitalet de Llobregat, Barcelona (V. Viñas-Díez, J. GascónBayarri, R. Reñé-Ramírez). Grupo de Investigación: Envejecimiento, Discapacidad y Salud; Institut $\mathrm{d}^{\prime}$ Investigació Biomèdica de Girona, IDIBGI; Girona (J.L. Conde-Sala, O. Turró-Garriga). Registro de Demencias; Institut d'Assistència Sanitària; Salt, Girona (O. TurróGarriga). Facultad de Psicología; Universitat de Barcelona; Barcelona, España (V. Viñas-Díez, J.L. CondeSala).

Correspondencia:

Dr. Josep Lluís Conde Sala. Facultad de Psicología. Universitat de Barcelona. Pg. Vall d'Hebron, 171. E-08035 Barcelona.

E-mail:

jllconde@ub.edu

Financiación:

Estudio financiado por el Ministerio de Economía y Competitividad del Gobierno de España en el marco del proyecto de investigación 'Evolución de la percepción de la calidad de vida del paciente, en pacientes con la enfermedad de Alzheimer y en sus familiares cuidadores, a lo largo de un período de dos años' (ref.: PSI2010-19014).

Aceptado tras revisión externa: 11.02.19.

Cómo citar este artículo: Viñas-Díez V, Conde-Sala JL, TurróGarriga O, Gascón-Bayarri J, ReñéRamírez R. Síntomas depresivos y sobrecarga en los familiares cuidadores en la enfermedad de Alzheimer: un modelo de ecuaciones estructurales. Rev Neurol 2019; 69: 11-7. doi: $10.33588 /$ rn.6901. 2018504.

c 2019 Revista de Neurología 
principal. Del mismo modo, Del Pino-Casado et al [25] expusieron que la presencia de alteraciones conductuales y la mayor dependencia funcional del paciente provocaban una situación de sobrecarga en el cuidador, con niveles de depresión más altos.

Respecto a los estudios longitudinales, se ha propuesto que una sobrecarga persistente predispone a padecer más síntomas depresivos en posteriores evaluaciones $[21,26]$, y que niveles más altos de depresión en el momento inicial predicen una mayor sintomatología depresiva en el seguimiento [27].

Nuestra hipótesis de partida fue que la sobrecarga tendría un efecto directo sobre la depresión. Los objetivos principales del estudio fueron observar la evolución de las características clínicas y sociodemográficas de pacientes y cuidadores, y aplicar un modelo de ecuaciones estructurales que clarificase la relación entre sobrecarga y sintomatología depresiva a lo largo de los dos años.

\section{Sujetos y métodos}

\section{Diseño y población de estudio}

Se realizó un estudio longitudinal con un seguimiento de 24 meses en una muestra consecutiva de familiares cuidadores de pacientes diagnosticados de probable enfermedad de Alzheimer según los criterios de la National Institute on Aging-Alzheimer's Association [28] y del Manual diagnóstico y estadístico de los trastornos mentales, cuarta edición, texto revisado (DSM-IV-TR) [29].

La muestra estuvo formada inicialmente por 221 pacientes y cuidadores, de los cuales 127 completaron el seguimiento. Todos los participantes fueron atendidos ambulatoriamente por la unidad de diagnóstico y tratamiento de las demencias del servicio de neurología del Hospital Universitari de Bellvitge. Todos los cuidadores fueron familiares de primer grado. Los criterios de exclusión para los pacientes fueron: presencia de problemas de comunicación graves, presencia de cualquier otra enfermedad neurológica o daño cerebral adquirido, y abuso de drogas o alcohol.

El estudio fue aprobado por el comité de ética de investigación clínica del hospital (ref. PR162/10). Tanto pacientes como cuidadores aceptaron participar mediante el consentimiento informado.

\section{Instrumentos}

- Datos sociodemográficos. Se recogieron mediante un cuestionario estructurado y diseñado ad hoc.
- Sobrecarga. Se utilizó el Caregiver Burden Inter$v i e w$ de Zarit [30]. La escala está formada por 22 ítems de respuesta de tipo Likert (de 1, nunca, a 5 , casi siempre), y el rango de puntuación oscila entre 22 y 110 puntos. A mayor puntuación, mayor sentimiento de carga.

- Depresión. Se utilizó la escala de depresión geriátrica [31] en su versión abreviada. Consta de 15 ítems dicotómicos, y se establece una puntuación $\geq 6$ para determinar una probable sintomatología depresiva y $\geq 9$ para determinar una depresión establecida.

- Estado de salud. Se administró al cuidador principal el Short Form-12 [32]. Es una versión abreviada del SF-36 Health Survey y valora la percepción de la salud del cuidador en dos dimensiones: mental y física. Consta de 12 ítems, y el rango de puntuación oscila entre 12 y 28 puntos. En las dos dimensiones, el rango es de 0 a 100. A mayor puntuación, mejor percepción de salud.

- Estado cognitivo. Se administró a los pacientes el Minimental State Examination [33]. Es un breve examen cognitivo con un rango de puntuación entre 0 y 30 . Para compensar la influencia de la edad y escolaridad, se aplicó la corrección para la población española [34]. Una menor puntuación implica un mayor deterioro cognitivo.

- Estado funcional. Se utilizó la escala Disability Assessment of Dementia [35] para evaluar la autonomía del paciente en las actividades de la vida diaria. Dicha escala está formada por 40 ítems, de los cuales 17 corresponden a las actividades de la vida diaria básicas y los otros 23 a las instrumentales. El rango de la puntuación oscila entre 40 y 80 puntos. A menor puntuación, mayor dependencia funcional.

- Sintomas psicológicos y conductuales. Se evaluaron mediante el inventario neuropsiquiátrico [36], según el criterio del cuidador principal. Está formado por 12 subescalas que valoran la frecuencia y la gravedad de los síntomas. El rango de puntuación va de 0 a 144 puntos. Una mayor puntuación corresponde a mayores síntomas neuropsiquiátricos.

\section{Procedimiento}

Pacientes y cuidadores principales fueron seleccionados por los neurólogos de la unidad de diagnóstico y tratamiento de las demencias. Todos fueron evaluados de forma individual por dos psicólogas expertas en la administración de los instrumentos. Se realizaron tres evaluaciones a lo largo del seguimiento (evaluación basal, a los 12 meses y a los 24 meses). 


\section{Análisis estadístico}

Para comparar las variables sociodemográficas y clínicas entre la evaluación basal y los 24 meses, se utilizó la prueba de chi cuadrado para las variables categóricas y la $t$ de Student para las cuantitativas.

Se realizaron análisis de regresión multivariante para identificar las variables clínicas o sociodemográficas que contribuían a la carga y a la depresión del cuidador, introduciendo todas las variables al mismo tiempo.

Con el fin de evaluar la relación de dependencia entre la sobrecarga y la depresión, se ajustó un modelo de ecuaciones estructurales, con las puntuaciones de las diferentes variables en el momento basal y a los 24 meses. El modelo conceptual se basó en una variación del desarrollado por Sherwood et al [24] y se muestra en la figura 1.

El método de estimación fue el de máxima verosimilitud. Se evaluó el modelo basándose en los estadísticos de bondad de ajuste: $\chi^{2}>0,05$, con grados de libertad < 3; Comparative Fit Index (CFI) $\geq 0,95$, Tucker-Lewis Index (TLI) $\geq 0,95$ y root mean square error of approximation (RMSEA) $<0,08$ [37]. El tamaño del efecto de los coeficientes estandarizados se valoró según los criterios de Kline [38]: pequeño $(<0,1)$, moderado $(0,3)$ y fuerte $(\geq 0,5)$.

El análisis se realizó mediante el programa estadístico SPSS y AMOS en su versión 22. Para los contrastes de hipótesis, el nivel de significación estadística considerado fue de $p<0,05$.

\section{Resultados}

\section{Datos sociodemográficos y}

clínicos de pacientes y cuidadores

En la evaluación basal, los casos perdidos $(n=94)$ de los pacientes tuvieron menor nivel cognitivo (17,2 frente a 19,$1 ; p=0,01$ ), mayor deterioro funcional $(54,7$ frente a 60,$6 ; p<0,001)$ y mayores síntomas neuropsiquiátricos (31,2 frente a 20,9; $p<0,001)$. En los cuidadores sólo fue significativa la mayor sobrecarga (52,5 frente a 47,3; $p=0,15)$.

La muestra final del estudio estuvo formada por un total de 127 díadas, con un seguimiento completo a los 24 meses. En la evaluación basal, la edad media de los pacientes fue de 76,6 años, hubo una mayor presencia de mujeres (63\%) y la mayoría tenía una escolaridad inferior a ocho años $(96,1 \%)$. En el grupo de cuidadores, la edad media fue de 63,7 años, con un mayor número de mujeres (62,3\%), y el 64,6\% tenía estudios superiores a ocho años.
Tabla I. Datos sociodemográficos de pacientes y cuidadores.

\begin{tabular}{|c|c|c|c|c|c|c|}
\hline & & \multirow{2}{*}{$\begin{array}{c}\text { Basal } \\
(n=127)\end{array}$} & \multirow{2}{*}{$\begin{array}{c}24 \text { meses } \\
(n=127)\end{array}$} & \multicolumn{3}{|c|}{ Diferencias } \\
\hline & & & & $t / \chi^{2}$ & $p$ & $d / V$ \\
\hline & Edad (años) a & $76,6 \pm 7,3$ & $79,0 \pm 7,2$ & 2,62 & $0,009^{b}$ & 0,33 \\
\hline \multirow[t]{3}{*}{ Pacientes } & Mujeres & $80(63 \%)$ & $80(63 \%)$ & & & \\
\hline & Escolaridad ( $\leq 8$ años) & $122(96,1 \%)$ & $122(96,1 \%)$ & & & \\
\hline & Edad (años) a & $63,7 \pm 11,9$ & $65,7 \pm 11,9$ & 1,34 & 0,181 & 0,16 \\
\hline \multirow[t]{2}{*}{ Cuidadores } & Mujeres & $79(62,3 \%)$ & $81(63,8 \%)$ & 0,68 & 0,795 & 0,02 \\
\hline & Escolaridad ( $\leq 8$ años) & $82(64,6 \%)$ & $82(64,6 \%)$ & & & \\
\hline
\end{tabular}

$d$ : $d$ de Cohen (tamaño del efecto): débil $(<0,5)$ moderado $(0,5-0,8)$ fuerte $(>0,8) ; t: t$ de Student; $V: V$ de Cramer. a Media \pm desviación estándar; ${ }^{b} p<0,05$

Figura 1. Modelo conceptual de la sobrecarga y la depresión.

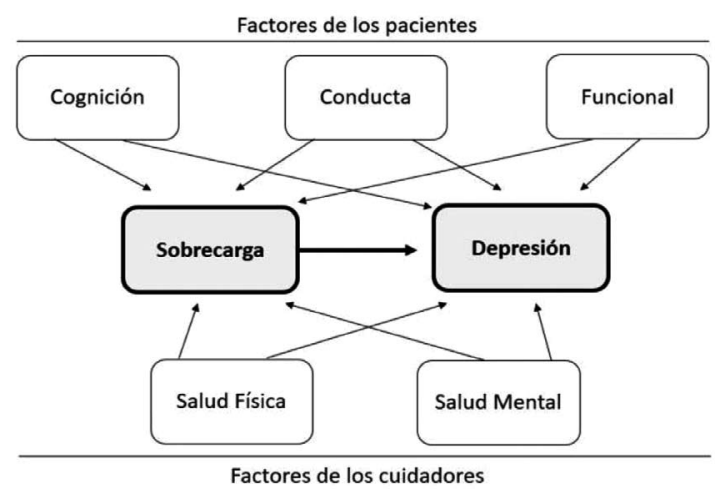

Factores de los cuidadores

En referencia a las variables clínicas, se observó que los pacientes presentaron un mayor deterioro cognitivo, físico y conductual a lo largo del seguimiento. Respecto a los cuidadores, se detectó un empeoramiento general (en salud física, mental y en depresión), aunque únicamente fue significativo el incremento de la sobrecarga a los 24 meses. Los datos se muestran en las tablas I y II.

\section{Análisis de regresión multivariante. Factores, sobrecarga y depresión}

Cuando se realizó el análisis multivariante, se observó que los factores que más contribuían a presentar niveles superiores de sobrecarga en el cuidador principal fueron las alteraciones conductuales y 
Tabla II. Datos clínicos de pacientes y cuidadores.

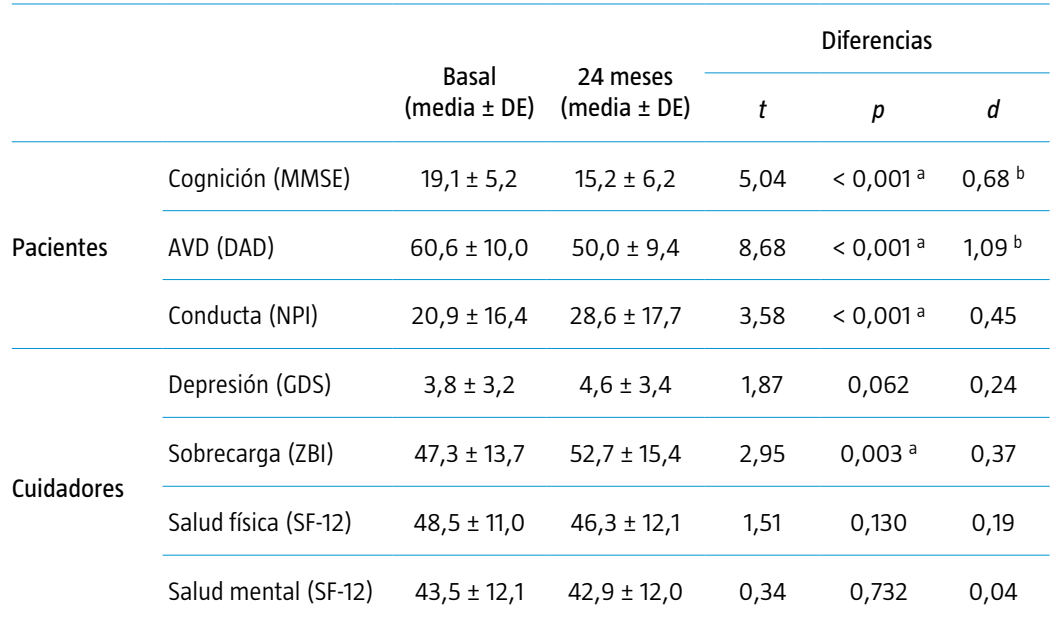

AVD: actividades de la vida diaria; $d$ : $d$ de Cohen, débil $(<0,5)$, moderado $(0,5-0,8)$ y fuerte $(>0,8)$; DAD: Disability Assessment for Dementia; DE: desviación estándar; GDS: Geriatric Depression Scale; MMSE: Minimental State Examination; NPI: Neuropsychiatric Inventory; SF-12: Short Form of Health Survey; $t$ : $t$ de Student; ZBI: Zarit Burden Inventory. a $p<0,05 ;$ b Tamaños del efecto moderados y fuertes.

la dependencia funcional del paciente; y la depresión y la salud mental del cuidador. Los factores que más contribuyeron a la presencia de sintomatología depresiva en el cuidador principal fueron la menor salud mental y física, la sobrecarga y la menor escolaridad del cuidador principal; y el menor nivel cognitivo de los pacientes.

Finalmente, se evidenció la existencia de una correlación moderada entre la depresión y la sobrecarga $(r=0,47)$ en la evaluación basal y fuerte $(r=$ $0,613)$ a los 24 meses. Los datos se muestran en la tabla III.

\section{Modelo de ecuaciones estructurales}

Basándonos en los datos del análisis de regresión, se diseñó un modelo de ecuaciones estructurales, con la sobrecarga y la depresión como variables dependientes. En relación con los factores de los pacientes, se observó que las alteraciones conductuales y el nivel funcional influyeron significativamente en la sobrecarga, en la evaluación basal y a los dos años. En cambio, el nivel cognitivo contribuyó a una mayor depresión en las dos evaluaciones y no tuvo ningún efecto directo sobre la sobrecarga.

Respecto a los factores de los cuidadores, se observó que una peor salud física sólo influyó en la depresión en el momento inicial. Una peor salud mental estuvo asociada a una mayor sobrecarga y depresión, tanto en el momento basal como a los dos años. La mayor escolaridad del cuidador se asoció de forma directa con la sobrecarga en el momento basal y de forma inversa a los dos años.

Los predictores de la sobrecarga explicaron el $50 \%$ de su varianza en el momento inicial y el $59 \%$ a los dos años, y los predictores de la depresión explicaron el 54\% de la varianza en el momento basal y el 70\% a los dos años. Los factores expuestos en el modelo se asociaron con unos niveles superiores de sobrecarga y depresión a los dos años, y destacó una mayor incidencia de la sobrecarga sobre la depresión en el seguimiento. Tanto la sobrecarga como la depresión iniciales predecían la persistencia de estas mismas variables a los dos años.

Según los criterios establecidos, se confirmó la bondad de ajuste del modelo $\left(\chi^{2}=1,144 ; \mathrm{gl}=1 ; p=\right.$ 0,285 ; RMSEA $=0,034$; NFI $=0,99$; TLI $=0,98$; CFI $=1,00)$, lo que corrobora el hecho de que todos los valores obtenidos eran aceptables. En la figura 2 se expone el modelo reflejando sólo las variables significativas.

\section{Discusión}

La asociación entre la sobrecarga percibida y la depresión se ha constatado en múltiples investigaciones [19-27], aunque existen discrepancias respecto a cómo se interrelacionan. Nuestro modelo final puso de relieve que la carga influía directamente sobre la sintomatología depresiva, y que, además, las características clínicas del paciente y del cuidador desempeñaban un papel importante en dicha relación. Estos hallazgos confirman nuestra hipótesis inicial y corroboran lo ya expuesto por estudios anteriores, donde se consideraba que niveles superiores de sobrecarga predisponían a padecer mayores síntomas depresivos en el cuidador [21-25].

Los factores del paciente que más contribuyeron a la sobrecarga, tanto inicialmente como a los dos años, fueron la presencia de síntomas neuropsiquiátricos y la dependencia funcional. Estos resultados apoyan investigaciones previas, donde se constató que estas variables están relacionadas con una mayor sobrecarga, que a su vez provocan una mayor depresión [24,25].

De acuerdo con investigaciones previas [14], el nivel cognitivo del paciente no afectó a la sobrecarga. Sin embargo, nuestro trabajo mostró que este factor sí contribuyó a la depresión tanto basal como a los dos años. En este sentido, Kim et al [16] ya refirieron que el deterioro cognitivo se relacionaba con un incremento de la depresión cuando los cuidadores eran los esposos y convivían con el pacien- 
te. Esta asociación podría deberse a los sentimientos de pérdida que experimenta el cuidador a medida que la enfermedad avanza y el paciente va perdiendo sus capacidades, y la relación habitual con el cuidador principal se modifica.

En relación con las características del cuidador, la peor salud mental contribuyó a presentar mayores niveles de sobrecarga y de depresión en las dos evaluaciones. En un estudio previo, Conde-Sala et al [12] sugirieron que el tratamiento de la salud mental del cuidador era uno de los elementos claves para la reducción de la carga, y que esto comportaría también una mejora de los niveles de depresión. Respecto a la salud física, Pinquart y Sörensen [18] habían resaltado su importancia, concluyendo que ésta se asociaba más fuertemente con los síntomas depresivos del cuidador que con las propias demandas del proceso de cuidado. Sin embargo, en nuestro estudio, el efecto de la salud física sólo fue significativo en la depresión basal.

Trabajos previos ponían de relieve que un bajo nivel de estudios se asociaba a mayores síntomas psicológicos $[15,17]$. En nuestro estudio, en el momento basal, niveles de educación superiores contribuyeron a presentar mayor sobrecarga, probablemente debido a que los cuidadores con más estudios eran más jóvenes y tenían más obligaciones laborales o familiares. En cambio, a los dos años, la mayor escolaridad se asoció con menor sobrecarga, posiblemente por una mayor comprensión de la enfermedad y la progresiva utilización de estrategias y recursos.

Por último, nuestro modelo confirma que la sobrecarga es un factor de riesgo potencial de la depresión [23-25], especialmente cuando ésta es persistente en el tiempo y predispone a padecer depresiones futuras [22,26]. Asimismo, de acuerdo con Vilchinsky et al [27], la existencia de síntomas depresivos en el momento basal también actúa como predictor de posibles depresiones posteriores.

\section{Conclusiones e implicaciones clínicas}

Dado que un elevado nivel de sobrecarga incide directamente en el riesgo del cuidador principal a padecer depresión, cabe destacar que la prevención o el tratamiento de dicha carga podrían disminuir los niveles de depresión. Una vez establecido el diagnóstico de demencia en el paciente, sería conveniente evaluar los factores relacionados con la carga del cuidador [39] para establecer intervenciones centradas en prevenirla y, en consecuencia, evitar la aparición de depresiones futuras. En este sentido, algunas investigaciones han concluido que una alta
Tabla III. Análisis de regresión multivariante.

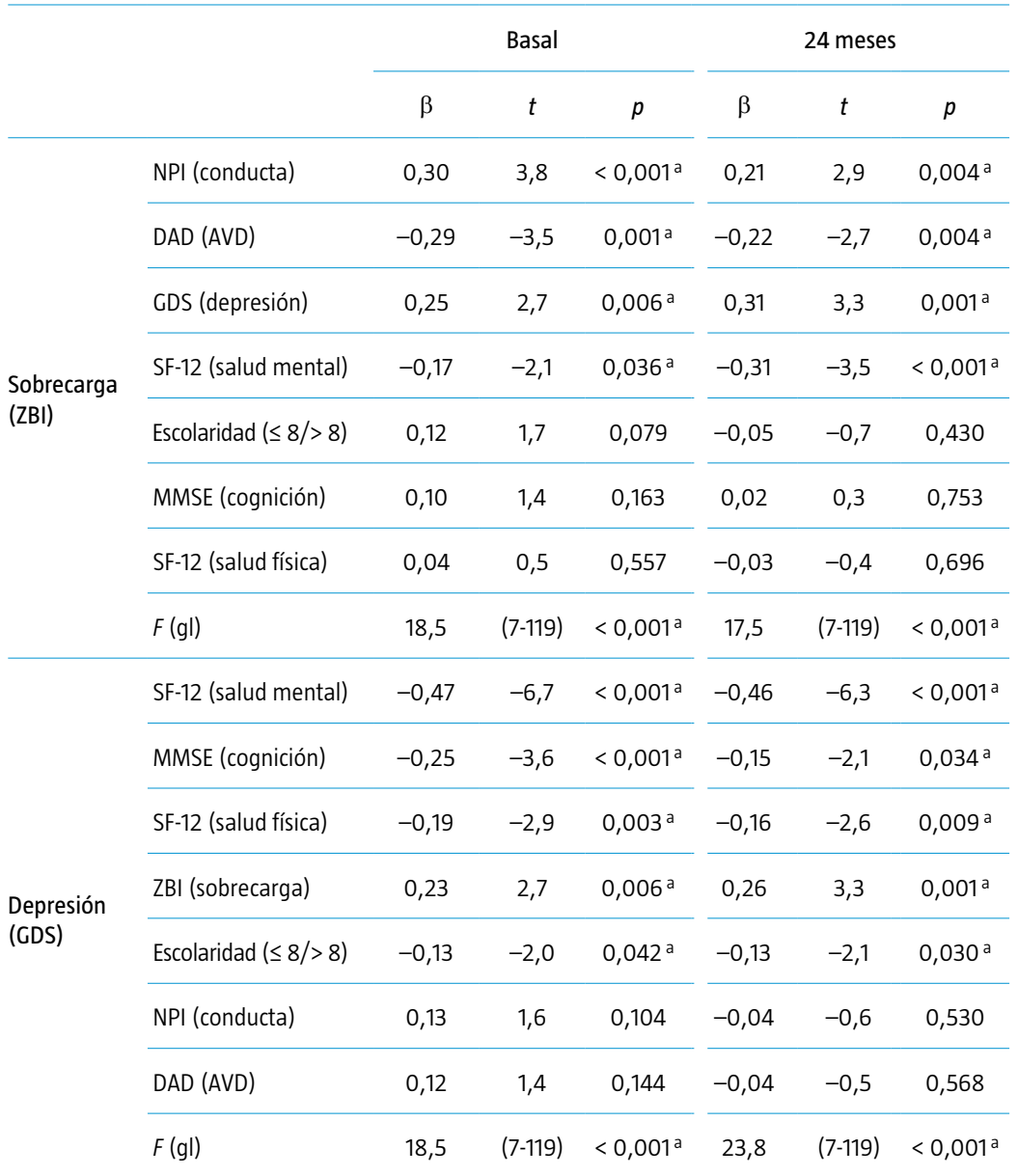

AVD: actividades de la vida diaria; $\beta$ : coeficiente estandarizado beta; DAD: Disability Assessment for Dementia; F: ANOVA; GDS: Geriatric Depression Scale; gl: grados de libertad; MMSE: Minimental State Examination; NPI: Neuropsychiatric Inventory; SF-12: Short Form of Health Survey; $t$ : $t$ de Student; ZBI: Zarit Burden Inventory. $R^{2}$ (coeficiente de determinación) =0,481 (sobrecarga basal); 0,508 (sobrecarga a 24 meses); 0,521 (depresión basal); 0,584 (depresión a 24 meses). a $p<0,05$.

satisfacción en el cuidado y bajos niveles de carga pueden generar efectos protectores de ansiedad y depresión en los cuidadores [40].

\section{Limitaciones e investigaciones futuras}

Una limitación asociada a los estudios longitudinales es la pérdida de casos en el seguimiento, por lo que es posible que muchos de los casos perdidos fueran pacientes en fases más avanzadas. Una segunda limitación hace referencia a que el análisis realizado ha sido global, sin tener en cuenta los casos en los que se produce un aumento o una disminución de la sobrecarga y la depresión. Este análisis requeriría un segundo estudio. 
Figura 2. Modelo de ecuaciones estructurales. Efectos directos estandarizados. ${ }^{*} p<0,05 ;{ }^{* *} p<0,01 ;{ }^{* * *} p<0,001$. Tamaño del efecto: pequeño $(<0,1)$, moderado $(0,3)$ y fuerte $(\geq 0,5)$.

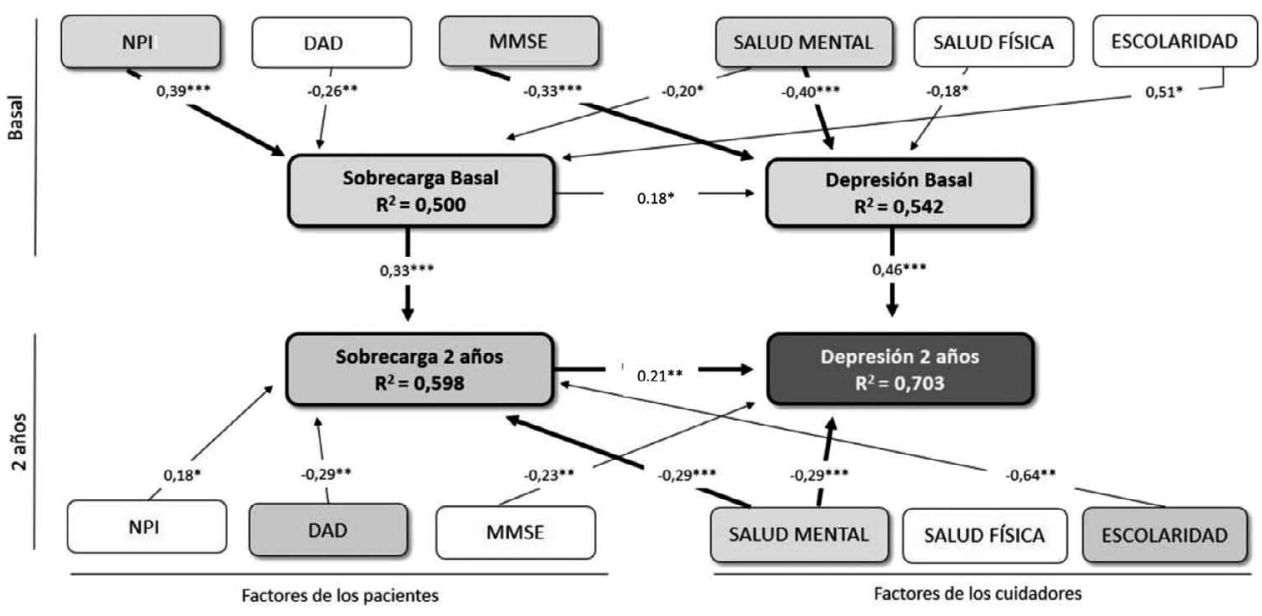

En investigaciones futuras sería interesante incluir la información relativa a los rasgos de personalidad del cuidador principal $[23,41]$ o las estrategias de afrontamiento utilizadas $[6,8]$, ya que pueden actuar como mediadores entre la carga y la depresión.

Finalmente, diversos estudios refieren la existencia de sentimientos positivos relacionados con el proceso de cuidado $[42,43]$, por lo que hubiera sido útil disponer de esta información para conocer si la satisfacción del cuidador principal podría disminuir la sobrecarga y, en consecuencia, la sintomatología depresiva.

\section{Bibliografía}

1. Garre-Olmo J. Epidemiología de la enfermedad de Alzheimer y otras demencias. Rev Neurol 2018; 66: 377-86.

2. Niu H, Álvarez-Álvarez I, Guillén-Grima F, Aguinaga-Ontoso I. Prevalencia e incidencia de la enfermedad de Alzheimer en Europa: metaanálisis. Rev Neurol 2017; 32: 523-32.

3. Peña-Longobardo LM, Oliva-Moreno J. Economic valuation and determinants of informal care to people with Alzheimer's disease. J Health Econ 2015; 16: 507-15.

4. Capistrant BD, Moon JR, Glymour MM. Spousal caregiving and incident hypertension. Am J Hypertens 2012; 25: 437-43.

5. Mausbach BT, Patterson TL, Rabinowitz YG, Grant I, Schulz R. Depression and distress predict time to cardiovascular disease in dementia caregivers. Health Psychol 2007; 26: 539-44.

6. García-Alberca JM, Cruz B, Lara JP, Garrido V, Lara A, Gris E. Anxiety and depression are associated with coping strategies in caregivers of Alzheimer's disease patients: results from the MÂLAGA-AD study. Int Psychogeriatr 2012; 24: 1325-34.

7. Conde-Sala JL, Garre-Olmo J, Turró-Garriga O, Vilalta-Franch J, López-Pousa S. Differential features of burden between spouse and adult-child caregivers of patients with Alzheimer's disease: an exploratory comparative design. Int J Nurs Stud 2010; 47: 1262-73.
8. García-Alberca JM, Cruz B, Lara JP, Garrido V, Gris E, Lara A et al. Disengagement coping partially mediates the relationship between caregiver burden and anxiety and depression in caregivers of people with Alzheimer's disease. Results from the MÁLAGA-AD study. J Affect Disord 2012; 136: 848-56.

9. Schulz R, McGinnis KA, Zhang S, Martire LM, Hebert RS, Beach SR, et al. Dementia patient suffering and caregiver depression. Alzheimer Dis Assoc Disord 2008; 22: 170-6.

10. Garre-Olmo J, Vilalta-Franch J, Calvó-Perxas L, Turró-Garriga O, Conde-Sala L, López-Pousa S. A path analysis of patient dependence and caregiver burden in Alzheimer's disease. Int Psychogeriatr 2016; 28: 1133-41.

11. Sousa MF, Santos RL, Turró-Garriga O, Dias R, Dourado MC Conde-Sala JL. Factors associated with caregiver burden: comparative study between Brazilian and Spanish caregivers of patients with Alzheimer's disease. Int Psychogeriatr 2016; 28: 1363-74.

12. Conde-Sala JL, Turró-Garriga O, Calvó-Perxas L, Vilalta-Franch J, López-Pousa S, Garre-Olmo J. Three-year trajectories of caregiver burden in Alzheimer's disease. J Alzheimers Dis 2014; 42: 623-33

13. Garre-Olmo J, López-Pousa S, Vilalta-Franch J, Turón-Estrada A, Hernández-Ferrándiz M, Lozano-Gallego M, et al. Carga del cuidador y síntomas depresivos en pacientes con enfermedad de Alzheimer. Evolución a los 12 meses. Rev Neurol 2002; 34: 601-7.

14. Van der Lee J, Bakker TJ, Duivenvoorden HJ, Droes RM. Multivariate models of subjective caregiver burden in dementia: a systematic review. Ageing Res Rev 2014; 15: 76-93.

15. Pattanayak RD, Jena R, Tripathi M, Khandelwal SK. Assessment of burden in caregivers of Alzheimer's disease from India. Asian J Psychiatr 2010; 3: 112-6.

16. Kim W, Lee TH, Shin J, Park E. Depressive symptoms in spouse caregivers of dementia patients: a longitudinal study in South Korea. Geriatr Gerontol Int 2017; 17: 973-83.

17. Omranifard V, Haghighizadeh E, Akouchekian S. Depression in main caregivers of dementia patients: prevalence and predictors. Adv Biomed Res 2018; 7: 34.

18. Pinquart M, Sörensen S. Correlates of physical health of informal caregivers: a meta-analysis. J Gerontol B Psychol Sci Soc Sci 2007; 62: 126-37.

19. De Fazio P, Ciambrone P, Cerminara G, Barbuto E, Bruni A, Gentile P, et al. Depressive symptoms in caregivers of patients 
with dementia: demographic variables and burden. Clin Interv Aging 2015; 10: 1085-90.

20. Springate BA, Tremont G. Dimensions of caregiver burden in dementia: Impact of demographic, mood, and care recipient variables. Am J Geriatr Psychiatry 2014; 22: 294-300.

21. Clyburn LD, Stones MJ, Hadjistavropoulos T, Tuokko H. Predicting caregiver burden and depression in Alzheimer's disease. J Gerontol B Psychol Sci Soc Sci 2000; 55: S2-13.

22. Epstein-Lubow G, Davis JD, Miller IW, Tremont G. Persisting burden predicts depressive symptoms in dementia caregivers. J Geriatr Psychiatry Neurol 2008; 21: 198-203.

23. Clark MC, Diamond PM. Depression in family caregivers of elders: a theoretical model of caregiver burden, sociotropy, and autonomy. Res Nurs Health 2010; 33: 20-34.

24. Sherwood PR, Given CW, Given BA, Von Eye A. Caregiver burden and depressive symptoms: analysis of common outcomes in caregivers of elderly patients. J Aging Health 2005; 17: 125-47.

25. Del-Pino-Casado R, Palomino-Moral PA, Pastor-Bravo MDM, Frías-Osuna A. Determinants of depression in primary caregivers of disabled older relatives: a path analysis. BMC Geriatr 2017; 17: 274.

26. Grano C, Lucidi F, Violani C. The relationship between caregiving self-efficacy and depressive symptoms in family caregivers of patients with Alzheimer disease: a longitudina study. Int Psychogeriatr 2017; 29: 1095-103.

27. Vilchinsky N, Dekel R, Revenson TA, Liberman G, Mosseri M. Caregivers' burden and depressive symptoms: the moderational role of attachment orientations. Health Psychol 2015; 34: 262-9.

28. McKhann GM, Knopman DS, Chertkow H, Hymanf BT, Jack CR, Kawash $\mathrm{CH}$, et al. The diagnosis of dementia due to Alzheimer's disease: recommendations from the National Institute on Aging-Alzheimer's Association workgroups on diagnostic guidelines for Alzheimer's disease. Alzheimers Dement 2011; 7: 263-9.

29. American Psychiatric Association. Diagnostic and statistica manual of mental disorders, DSM-IV-TR. Washington DC: APA Press; 2000.

30. Zarit SH, Todd PA, Zarit JM. Subjective burden of husbands and wives as caregiver: a longitudinal study. Gerontologist 1986; 26: 260-6.
31. Sheikh JI, Yesavage JA. Geriatric Depression Scale (GDS): recent evidence and development of a shorter version. Clin Geront 1986; 5: 165-73.

32. Ware JJr, Kosinski M, Keller SD. A 12-Item Short-Form Health Survey: construction of scales and preliminary tests of reliability and validity. Med Care 1996; 34: 220-33.

33. Folstein MF, Folstein SE, McHugh PR. 'Mini Mental State' A practical method for granding the cognitive state of patients for the clinician. J Psychiatr Res 1975; 12: 189-98.

34. Blesa, R, Pujol M, Aguilar M, Santacruz P, Bertrán-Serra I, Hernández G, et al. Clinical validity of the mini-mental state for Spanish speaking communities. Neuropsychologia 2001; 39: 1150-7.

35. Gélinas I, Gauthier L, McIntyre M, Gauthier S. Development of a functional measure for persons with Alzheimer's disease: the Disability Assessment for Dementia. Am J Occup Ther 1999; 53: 471-81.

36. Cummings JL, Mega M, Gray K, Rosemberg-Thompson S, Carusi DA, Gornbein J. The neuropsychiatric inventory. Comprehensive assessment of psychopathology in dementia. Neurology 1994; 44: 2308-14.

37. Ruiz MA, Pardo A, San Martín R. Modelos de ecuaciones estructurales. Papeles del Psicólogo 2010; 31: 34-45.

38. Kline RB. Principles and practice of structural equation modeling. 3 ed. New York: Guilford Press; 2011.

39. Adelman RD, Tmanova LL, Delgado D, Dion S, Lachs MS. Caregiver burden. A clinical review. JAMA 2014; 311: 1052-60.

40. Del Pino-Casado R, Palomino-Moral PA, Fras-Osuna A. The association of satisfaction and perceived burden with anxiety and depression in primary caregivers of dependent elderly relatives. Res Nurs Health 2015; 38: 384-91.

41. Melo G, Maroco J, De Mendonça A. Influence of personality on caregiver's burden, depression and distress related to the BPSD. Int J Geriatr Psychiatry 2011; 26: 1275-82.

42. Bastawrous M, Cignac MA, Kapral MK, Cameron JI. Factors that contribute to adult children caregivers' well-being: a scoping review. Health Soc Care Community 2015; 23: 449-66.

43. Chappell NL, Dujela C, Smith A. Spouse and adult child differences in caregiving burden. Can J Aging 2014; 33: 462-72.

\section{Depressive symptoms and burden in family caregivers in Alzheimer's disease: a model of structural equations}

Introduction. The care of patients with Alzheimer's disease increases the burden and depression of the main caregiver, although the relationship between the two is not clearly defined.

Aims. To explore the factors associated with depressive symptomatology and burden in the main caregiver and to apply a model of structural equations to identify the relationship between them.

Subjects and methods. The sample consisted of 127 family caregivers of people with Alzheimer's disease, with a follow-up of 24 months. Multivariate regression analyses were performed to identify the characteristics of patients and caregivers associated with burden and depression of the main caregiver, and a model of structural equations was designed to analyse the relationship between the variables.

Results. In the model of structural equations, the burden had a direct effect on depression, both in the baseline assessment and at 24 months. The variables associated with burden were: schooling and the lower mental health of the main caregiver; and the behavioral alterations and the functional dependency of the patient. The lower cognitive level of the patient and the lower mental health of the main caregiver were associated with depression. The correlation between burden and depression increased from baseline to two years ( $r=0.470$ vs. $r=0.613$ ).

Conclusions. The deterioration of the patient and caregiver burden are risk factors for depression in the main caregiver. Interventions would be necessary to reduce the burden and prevent related depression.

Key words. Alzheimer. Burden. Caregiver. Depression. Mental health. Structural equations. 